\title{
Ispahan, moitié du monde. Dimension géocritique de l'espace dans Vers Ispahan de Pierre Loti et L'Usage du monde de Nicolas Bouvier
}

\author{
Safoura Tork Ladani \\ Université d'Ispahan
}

\section{Introduction}

L'espace en tant que thème de prédilection dans les recherches littéraires a été abordé ces dernières décennies dans la littérature comparée, notamment dans l'approche géocritique qui offre une manière d'appréhender les lieux étudiés comme des espaces imaginaires. Cette approche consiste à analyser les représentations des espaces géographiques chez les écrivains ou voyageurs d'une période précise.

En fait, la géocritique trouve ses racines dans les idées de Deleuze, mais surtout de Gaston Bachelard qui donne une autre définition de l'espace qui révèle une autre dimension de cette notion. Chez lui, l'espace « est vécu avec toutes les particularités de l'imagination» (Bachelard 17). Pour Bertrand Westphal, la géocritique envisage les espaces humains qui ont pris une dimension imaginaire dans les ouvrages littéraires (8). Alors, cette approche est fondée sur un va-et-vient entre le lieu réel et le lieu imaginaire. Selon Westphal, la géocritique se définit comme une «approche littéraire (ou interartistique) de la représentation des espaces humains » (8). Il s'efforce de donner une vision différente du regard sur l'Autre et sur l'Ailleurs. Selon lui, « il s'agira de sonder les espaces humains que les arts mimétiques agencent par et dans le texte, par et dans l'image, ainsi que les interactions culturelles qui se nouent sur leur patronage » (17). Cela sera réalisé par le biais d'une démarche dont les principaux éléments sont la multifocalisation, la polysensorialité, la stratigraphie et l'intertextualité.

La multifocalisation signifie la représentation d'un lieu réel à travers différents points de vue. En fait, la géocritique cherche à éclairer la sensibilité propre à chaque écrivain par rapport à un lieu décrit. Selon Westphal, «si cette critique est confinée à l'étude d'un seul texte, ou d'un seul auteur, il devient bancal» (205).

La polysensorialité envisage le texte comme un recueil de signes sensoriels qui rendent spécial un lieu ou un objet décrit. Effectivement, ce sont les expériences liées aux sens qui valorisent ce lieu ou cet objet :

L'expérience est un mot-valise qui comporte les différents modes par lesquels une personne connaît et bâtit la réalité. Ces modes intègrent aussi bien les sens les plus directs et les plus passifs, comme l'odorat, le goût et le toucher que la perception visuelle active et le mode indirect de la symbolisation (Tuan 54). 
Du point de vue de la stratigraphie, l'architecture de chaque monument et la culture des habitants de chaque ville révèlent l'horizon de son passé. Selon Westphal, «avec la littérature et l'espace, nous n'avons pas affaire à des choses en soi, mais à des objets culturels qui ont leur géographie et leur âge, celles de la configuration d'axiomes sur laquelle repose la science contemporaine et que Michel Foucault appelle notre épistémè » (11).

Le quatrième élément de la géocritique westphalienne est l'intertextualité. Dans bien des ouvrages littéraires, nous pouvons rencontrer l'influence d'autres textes littéraires, soit consciemment soit inconsciemment.

Dans cet article, nous avons choisi la ville d'Ispahan, une ville située au centre de l'Iran. En nous appuyant sur la géocritique westphalienne, nous ferons une analyse comparative de l'image donnée de cette ville à des époques différentes par les deux voyageurs, Pierre Loti et Nicolas Bouvier. Pierre Loti, voyageur français du XIX ${ }^{\mathrm{e}}$ siècle, à l'époque Qadjar, a décrit la ville dans son ouvrage Vers Ispahan; Nicolas Bouvier, le voyageur suisse, a parcouru l'Iran au $\mathrm{XX}^{\mathrm{e}}$ siècle, à l'époque pahlavi et a livré ses observations dans son ouvrage intitulé L'Usage du monde.

Dans cette étude, nous allons examiner comment Pierre Loti et Nicolas Bouvier, tous deux voyageurs francophones, mais issus de deux nations et époques différentes, ont décrit la ville d'Ispahan, chacun à sa manière, selon la mentalité et l'expérience propres à chacun. Une première mentalité qui s'imprègne des stéréotypes des siècles précédents de «l'orient merveilleux », «la Perse des mille et une nuits» et une autre qui profite d'une ambiance moderne du $\mathrm{XX}^{\mathrm{e}}$ siècle.

\section{1) La géocritique et les récits de voyage}

C'est avec l'approche du lieu que se réalise l'approche géocritique. Les récits de voyage sont «incontestablement, des espaces discursifs féconds pour l'étude de la spatialité.» (Outeirinho 153). Le voyageur n'est pas seulement un observateur passionné par l'exotisme, mais, quelque chose en plus qui dépasse les frontières et les limites.

On a néanmoins continué à écrire et décrire des espaces autres. Malgré la relativisation grandissante du concept d'exotisme, le récit de voyage s'est perpétué. Peut-être même a-t-il continué à s'affirmer. Le voyageur ne se cantonne plus dans le seul spectacle sensible du monde. Il rend compte de la qualité abstraite des espaces qu'il parcourt. (Westphal 46).

Le récit de voyage relate les déplacements du voyageur dans l'espace et offre, par la suite, des informations personnelles sur l'espace traversé. En fait, l'un des traits importants de ce type de récit est que l'auteur y transmet tout ce qu'il voit et expérimente dans l'espace qu'il traverse, « car le récit de 
voyage s'érige à partir d'une expérience de l'espace et de la représentation de cette expérience : la littérature de voyage présente une lecture du monde et la clé de lecture de ce voyageur-narrateur particulier. » (Outeirinho 153)

\section{1) La multifocalisation : une image multipliée d'Ispahan}

Dans la géocritique de Westphal, nous sommes toujours confrontés à la multiplicité des focalisations ou la multiplicité des points de vue. Selon lui «toute approche géocritique à travers l'étude d'un seul texte ou d'un seul auteur serait périlleuse »(213), parce que dans ce cas, cette approche ressemble à une critique purement imagologique ${ }^{1}$.

La multifocalisation se base sur trois points de vue : les points de vue endogène, exogène et allogène. Le premier est une vision familière de l'espace, complètement distincte de l'exotisme. Le second marque la vision du voyageur née de l'exotisme. Ce point de vue est à la base du récit de voyage, celui sur lequel notre attention se focalise dans cet article. Quant au point de vue allogène, il se situe entre le premier et le second. Il se concentre sur un lieu qui n'est ni familier ni exotique.

Pour nos deux auteurs-voyageurs, il faut mentionner que le décalage du temps entre leurs voyages était d'environ soixante ans. Le premier voyageait au sein d'une caravane alors que le second voyageait avec son ami peintre, Thierry Vernet. Le but de l'auteur de Vers Ispahan est de visiter cette ville centrale de l'Iran en donnant des descriptions plus détaillées. C'est pourquoi il commence son ouvrage par la description de cette ville et invite son lecteur à l'accompagner dans ce voyage.

Dans le prélude de son récit de voyage, Loti présente une image globale qui est pour lui la " ville des roses », " la vieille ville de ruines et de mystère », la ville «des dômes bleus » et des «minarets bleus d'un inaltérable émail. » (2). Ce qui marque l'aspect fascinant d'une «Perse merveilleuse » des récits de voyage de Chardin et d'autres voyageurs occidentaux qui ont visité la Perse dans les siècles précédents.

Dans cette première description, il parle des « interminables plaines aussi haut montées que fleurettes pâles », de « quelque village, d'un gris tourterelle, avec sa petite mosquée croulante, au dôme plus adorablement bleu qu'une turquoise » et « des fantômes de palais » (3).

${ }^{1}$ L'imagologie, en tant qu'approche de la littérature comparée, étudie l'image des étrangers dans une œuvre littéraire ou un récit de voyage. Cette approche se focalise sur l'image de l'Autre et s'intéresse à l'Altérité pour bien analyser le regard d'un regardant qui pourrait être un voyageur ou un écrivain-voyageur sur un regardé qui se manifeste parfois sous forme d'un peuple ou d'un lieu. Par exemple, l'étude de l'image donnée de la Perse et des Persans par les voyageurs français du grand siècle, tels Chardin ou Tavernier, fait partie de cette méthode de recherche. 
L'Usage du monde de Bouvier n'est pas consacré complètement à la description de cette ville. Au contraire, Bouvier n'invite pas ses lecteurs à découvrir cette ville. Il parle plutôt de voyage, et il insiste d'ailleurs sur cette phrase : «On croit qu'on va faire un voyage, mais bientôt c'est le voyage qui vous fait, ou vous défait » (13).

\section{2) La polysensorialité : mélange des sensations sur Ispahan}

Le texte écrit sur une ville est une sorte d'outil de communication sensorielle créé par l'auteur pour rendre cette ville spéciale. Ainsi, les sens sont mis en scène de manière cohérente pour donner une identité polysensorielle à la description d'une ville dans un récit de voyage et faire vivre l'expérience du voyageur qui visite ce lieu. En fait, une expérience liée aux sens ne s'oubliera jamais, car les sens valorisent la ville décrite et la rendent séduisante et inoubliable.

Parmi les cinq sens (vue, ouie, goût, toucher et odorat), la vue est utilisée abondamment. Ce qui intéresse toujours le visiteur, c'est la couleur. Chaque couleur provoque un sentiment chez les lecteurs et les couleurs prennent alors des aspects symboliques. En fait, la couleur est l'élément le plus fort dans une création imaginaire et picturale et c'est ici qu'on constate que chacun de nos écrivains-voyageurs essaie de la décrire d'après ses expériences, ses réflexions, ses sentiments, ses souvenirs, sa mentalité et son style.

L'étude polysensorielle proposée par la géocritique, appuyée sur les sensations visuelles, auditives et olfactives puisées par le descripteur dans l'espace réel et référentiel, contribue à la création d'un espace imaginaire dans le monde littéraire. Ainsi, la représentation d'Ispahan dans les deux récits de voyage n'est pas basée seulement sur la sensation visuelle, mais sur un mélange de diverses sensations qui organisent un lien fort entre le monde littéraire et les états d'âme du descripteur du monde réel.

Dans les propos de Loti, on constate une grande fascination pour la nature, les monuments, les mosquées, les ruelles, les maisons, etc. Pour les décrire, il recourt en premier lieu au sens de la vue : «Une heure de route, dans un sinistre petit désert, aux ondulations d'argile brune, qui sans doute est placé là pour préparer l'apparition de la ville d'émail bleu, et de sa fraîche oasis » (109).

On observe que l'image de «la ville d'émail bleu », c'est la perspective de cette ville orientale qui apparaît de loin aux yeux de Loti; car ses mosquées sont bleues. Loti continue sa description et présente une image des alentours : «Les larges fleurs blanches », « une puissante mêlée d'arbres, des peupliers, des saules, des yeuses, des platanes », «des dômes bleus » et «les minarets bleus d'Ispahan ». Enfin, une « ville bleue », une « ville de turquoise 
et de lapis » dans « un bois vert », c'est l'image poétique donnée d'Ispahan par Loti. Cette ville bleue lui semble «invraisemblable et charmante», apparaissant de loin comme « un vieux conte oriental » (ibid.). L'imaginaire s'entremêle alors avec le réel.

Il porte beaucoup d'attention à l'architecture des monuments historiques tels que les mosquées, les bazars, la place royale, les palais et bien d'autres lieux de cette ville. Ces descriptions sont souvent métaphoriques et littéraires.

Les myriades de petites coupoles en terre rosée sont là aussi parmi les branches. Mais tout ce qui monte un peu haut dans le ciel, minarets sveltes et tournés comme des fuseaux, dômes tout ronds, ou dômes renflés comme des turbans et terminés en pointe, portiques majestueux des mosquées, carrés de muraille qui se dressent percés d'une ogive colossale, tout cela brille, étincelle dans des tons bleus, si puissants et si rares que l'on songe à des pierres fines, à des palais en saphir, à d'irréalisables splendeurs de féerie. (ibid.)

Loti présente ailleurs une description plus complète de la mosquée impériale. «La porte gigantesque » de cette mosquée l'attire « comme l'entrée magique d'un gouffre bleu!» (121). Pourtant, il y a aussi d'autres couleurs qui marquent cet aspect exotique d'un monde étrange : "Les murailles intérieures merveilleusement brodées d'émaux bleus, verts, jaunes et blancs. » (122) Ici, il nous est impossible de représenter toutes les descriptions visuelles données par Loti des mosquées, des bazars, des maisons, des places, des avenues et de la nature de cette ville.

Chez Bouvier, le sens de la vue apparaît en premier lieu :

Avec un ressort arrière brisé, traversé lentement le canton agricole qui entoure la ville. Le soleil se couchait derrière de hauts platanes solitaires dont l'ombre oblique s'étendait sur des villages de terre aux douces arêtes rongées. Dans les champs de blé coupé, les gerbes retenaient la lumière et brillaient comme du bronze. Des buffles, des ânes, des chevaux noirs, et des paysans aux chemises éclatantes travaillaient à finir les moissons. On voyait le bulbe léger des mosquées flotter sur la ville étendue. Assis sur le capot pour soulager la voiture malade, assommé de fatigue, je cherchais un mot pour m'approprier ces images, et je me répétais machinalement : Carabas. (631)

Il décrit en détail les platanes, les champs de blé, des buffles, des animaux et il nomme toutes ces images enfin «Carabas » une notion que nous allons expliquer plus loin.

Bouvier arrive à Ispahan à la saison des moissons alors que Loti y était arrivé à celle des roses. Comme Loti, il voit les mosquées de loin. Ailleurs, il décrit la mosquée royale, avec « d'irremplaçables carreaux de faïence » et « ce fameux bleu », « coupé d'un peu de turquoise, de jaune et de noir» donne un « pouvoir de lévitation » qu'on lie «à la sainteté ». «L'immense coupole qui en est couverte tire vers le haut comme un ballon captif.» (638) Ici, comme chez Loti, ce sont les couleurs des mosquées, surtout le fameux bleu qui attirent l'attention de Bouvier. Une couleur exotique, un bleu persan. 
Loti a utilisé d'autres sensations que la vue qui est omniprésente dans toutes les descriptions de Vers Ispahan: «Et le parfum des roses du jardin, mêlé aux senteurs d'on ne sait quelles essences de harem dont les tentures sont imprégnées...» (114). Ici, Loti utilise sa sensation olfactive. C'est au printemps que Loti est entré dans cette ville et « c'est l'époque de la cueillette pour composer les parfums ». Et il y a « des montagnes de roses par terre » (112) dans les jardins.

En outre, Loti use de sa sensation auditive. Il décrit la musique qui est jouée dans cet espace lors de la levée du soleil, avec l'adjectif « sauvage » et compare «les sons de trompes » aux «beuglements d'une bête primitive" (133). Il s'efforce ainsi de mépriser ce type de musique persane ou peut-être il exprime simplement son idée ou son sentiment à propos de ces sons qu'il entend dans une ville orientale et étrange à ses yeux. Ailleurs, il mêle les trois sens (vue, odorat et ouie) pour décrire Ispahan dans la nuit:

Les roses embaument dans la nuit, les roses du jardin très muré et défendu sur lequel mon logis s'ouvre. On n'entend venir aucun bruit du dehors, puisque personne ne circule plus ; aucun roulement, puisqu'il n'existe point de voiture ; l'air limpide et sonore ne vous apporte de temps à autre que des sons de voix, tous glapissants, tous tristes ; appels chantés des muezzins, longs cris des veilleurs de nuit qui se répondent d'un quartier fermé à un autre, aboiements des chiens de garde, ou plaintes lointaines des chacals. Et les étoiles scintillent étrangement clair (125).

Bouvier recourt aussi à sa sensation auditive pour décrire la musique chez les Iraniens lorsqu'une belle musique persane de $\operatorname{tar}^{3}$ résonne à la radio. Selon Bouvier, la musique persane est belle comme « celle d'un Segovia détaché de tout » et ressemble à « un peu de verre brisé qui dégringole avec indolence. » (634). Ispahan se manifeste donc chez Loti comme une ville féérique et imaginaire, une ville bleue, marquée par la turquoise. D'ailleurs, elle apparait pour lui comme une triste oasis et une vieille ville pleine de ruines et de mystères, une ville "désirée », "invraisemblable et charmante autant qu'un vieux conte oriental», qui était autrefois « un des centres de la magnificence et du luxe sur la Terre. » (109). Ispahan visitée et décrite par Loti est une ville des temps passés qui porte en elle les signes d'une longue histoire qui a vécu des bas et des hauts, ce qui se manifeste à travers ses ruines.

Mais un aspect sacré marque bien une relation entre le naturel et le spirituel : «Une ville silencieuse, une ville sainte, ville de grands arbres et des fleurs », la ville des « roses » (112). Mais c'est une ville qui est entourée avec ses ruelles labyrinthiques au milieu du désert qui donne une autre image comme celle de « la ville des ruines et de silence » et « un Ispahan de ruines et de mort » qui est aussi entourée de solitude. C'est la représentation d'une ville étrange et des déserts », où Loti sent une sorte de " nostalgie du passé. » (ibid.). Dans la nuit, Ispahan est une autre peinture orientale, fantastique dont

${ }^{2}$ Ici, « primitive » veut dire « sauvage, brutale, féroce ».

${ }^{3}$ «Tar» est un instrument de la musique persane et turque. Ce mot persan signifie « Corde $»$. 
Loti esquisse « une peinture transparente sur un vitrail » (ibid. 140). Et enfin, lorsqu'il va quitter la ville, il jette ces mots : «[...] la ville aux dômes bleus, la ville aux ruines couleur tourterelle, s'est évanouie derrière nous. » (146). C'est ce qui marque sa vision de l'instabilité du monde, son aspect passager et le sentiment qui en sort d'une manière inconnue.

La démarche de Bouvier est complètement différente: il l'a visitée presque soixante ans plus tard, à l'époque moderne qui marque le début du modernisme persan. C'est pourquoi sa vision change, en fonction des conditions sociales différentes et de l'évolution de la société. Cette image est complètement différente du stéréotype d'une «Perse merveilleuse » chez

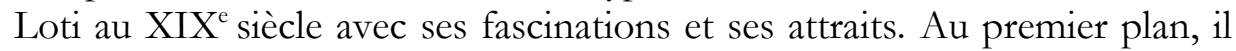
désigne les alentours de la ville, comme on a signalé, par le mot «Carabas » (631). Mais que signifie ce mot? Perrault reprend la définition de ce mot donnée dans la Bibliothèque orientale d'Herbelot de Molainville en 1697. Selon cette définition, Carabas est dérivé du mot turc Carabag, qui désigne les «montagnes [...] dans lesquelles il y avait autrefois des lieux de délices où les sultans mongols et autres princes faisaient leur séjour pendant l'été. » (135). Selon Bischoff, « le mot Carabas connote ainsi l'Orient, le dépaysement et l'appropriation de l'espace. » (53). Ce mot désigne un lieu avec ses spécificités orientales, un espace exotique qui attire de plus en plus le voyageur occidental, assoiffé de savoir sur l'Ailleurs. Ce mot symbolise en fait «la tentative d'approbation toujours fragile, par le voyageur, de nouveaux espaces qui ne lui appartiennent pas tant qu'il ne les a pas arpentés "par la plante des pieds", pour reprendre une expression chère à Nicolas Bouvier » (ibid.).

Si on compare les manières de décrire le côté effrayant d'Ispahan de Loti et de Bouvier, on constate que l'image donnée par Bouvier est plus forte et plus obscure. Lors de son entrée dans la ville, et après ses promenades, il trouve même qu'Ispahan est imprégnée du sentiment de la mort:

Mais une Provence sans vin, ni vantardises ni voix de femmes; en somme, sans ces obstacles ou ce fracas qui d'ordinaire nous isole de la mort. Je ne m'étais pas plutôt dit cela que j’ai commencé à la sentir partout, la mort : les regards qu'on croisait, l'odeur sombre d'un troupeau de buffles, les chambres éclairées béant sur la rivière, les hautes colonnes de moustiques. Elle gagnait sur moi à toute allure. Ce voyage? Un gâchis... un échec. (641)

Ici, «la narration va d'instants d'éblouissement, de véritables “épiphanies laïques, à de 'brusques effondrements de sens' (Cogaz 179). La perte est d'abord celle non pas de la durée, mais de l'ancrage au réel, 'cette ville impalpable, ce fleuve qui n'aboutit nulle part et d'ailleurs peu propre à vous asseoir dans le sentiment du réel”.

En fait, le sentiment provoqué chez Bouvier et Loti durant la visite de ces différents monuments, illustre bien la mentalité occidentale qui s'exerce tout au long de leur voyage. 
Influencés par cette altérité persane, ils s'efforcent de s'habituer à cette atmosphère bien étrange. Avant d'entrer dans cette ville, Loti se heurte à "la monotonie terreuse du désert". Après le désert, apparaissent les champs verts et la "verdure" de cette région est selon lui "plus exubérante encore que chez [eux]" et "elle est étonnamment verte" (109). Cette vision change selon l'aspect exotique de cette région où se trouve le désert et la verdure, collision de contradictions!

Le voyageur marque également la différence entre la ville orientale visitée et la ville occidentale qu'il a vue dans son pays. La citoyenneté et les conditions exigées à chaque espace vont former chez le voyageur une vision bien spécifique. Ainsi, Loti fait une comparaison entre les abords silencieux de la ville d'Ispahan et les alentours d'une grande ville française :

Ispahan! ... Mais quel silence aux abords! ... Chez nous, autour d'une grande ville, il y a toujours des kilomètres de gâchis enfumé, des charbons, de tapageuses machines en fonte, et surtout des réseaux de ces lignes de fer qui établissent la communication affolée avec le reste du monde. Ispahan, seule et lointaine dans son oasis, semble n'avoir même pas de routes. (ibid.)

La première avenue visitée par Loti est une "avenue large et droite". De chaque côté de cette avenue,

d'épais buissons de roses forment bordure; derrière ce sont des jardins où l'on aperçoit, parmi les arbres centenaires, des maisons ou des palais, en ruines peutêtre, mais on ne sait trop, tant la feuillée est épaisse. Ces massifs de rosiers en pleine rue, que les passants peuvent fourrager, ont fleuri avec une exubérance folle (112).

Mais comme les Iraniens nomment Ispahan "la moitié du monde" (Esfahân nesf-e-jahân), on observe que le voyageur est fasciné par un sentiment d'exaltation devant cette splendeur éternelle de la ville. Donc, on la nomme "la moitié" au lieu de "le milieu" du monde pour bien décrire cet aspect fascinant.

Ispahan "est inscrite au firmament de la mémoire de ceux qui y ont découvert la lumière de l'aube sur l'esplanade des mosquées, les mélopées des poètes au crépuscule, les ponts aux multiples arcades, le trépidant bazar." (Rostkowski 2). Ce qui marque Bouvier, c'est un "fameux bleu, coupé d'un peu de turquoise, de jaune et de noir qui le font vibrer et lui donnent ce pouvoir de lévitation qu'on n'associe d'ordinaire qu'à la sainteté." (233)

Au contraire de cette description fascinante de cette ville donnée par Bouvier, Loti, poète sensible et soucieux, regarde d'une autre manière. Il entre dans la ville par un chemin : "l'enfermement, l'oppression des ruines et du mystère nous attendaient au premier détour du chemin". Il croit que les maisons d'Ispahan ont de grands murs sans fenêtres comme celles de Chiraz et leurs portes sont fermées aux étrangers. Les ruelles sont désertes 
"avec des immondices par terre, des carcasses, des chiens morts". Alors, "tout est inhabité, caduc et funèbre" (109).

Alors, l'agitation qu'il voit dans le bazar après les abords et les ruelles déserts et silencieux de cette ville, lui parait pittoresque. Selon lui, "c'est un monde, les bazars d'Ispahan!" (ibid.)

Nicolas Bouvier donne une image précieuse de la mosquée la plus célèbre d'Ispahan, un tableau bien distingué du "porche d'émail bleu de la mosquée célèbre dans tout l'Islam." (176). C'est par sa sensation visuelle qu'il décrit cette mosquée dont "l'émail bleu" évoque le calme et la sérénité d’un lieu islamique. Cette couleur montre le ciel qui suggère Dieu et la spiritualité.

Loti visite la Place Impériale où se trouve cette mosquée et il la considère comme la merveille de la ville. Il dit plus tard : "Cette place d'Ispahan [...] n'a d'égale dans aucune de nos villes d'Europe, ni comme dimensions, ni comme magnificence." (119)

Il déclare tout en s'approchant de cette mosquée: “je m’approche au moins de la mosquée Impériale, dont la porte gigantesque, tout là-bas, m'attire comme l'entrée magique d'un gouffre bleu!' (ibid.) Ensuite, il commence à décrire minutieusement tout ce qu'il voit dans cette zone sacrée et d'une manière défendue, car les étrangers ne pouvaient pas y entrer. Enfin, il conclut: "L'ensemble de cela, qui est indescriptible d'enchevêtrement et de magnificence, dans des couleurs de pierreries, produit une impression d'unité et de calme, en même temps qu'on se sent enveloppé là de frâiche pénombre." Il croit que la porte de ce lieu sacré et islamique est "impénétrable pour les chrétiens." Cette porte "a l'air de s'enfoncer dans le royaume du bleu absolu et suprême." (122)

Mais Nicolas Bouvier, qui avait passé quelques instants à la mosquée en compagnie de ses amis iraniens, parle d'un sentiment produit par la prière, une sorte de "candeur" qui lui donne une extase, d'une conception de Dieu très proche et omniprésent auquel il pouvait "tout demander" (153), ce qui est proche de la vision poétique et métaphysique de Loti dans la description des lieux.

\section{3) La stratigraphie : Ispahan d'autrefois et Ispahan d'aujourd'hui}

Dans le présent de toute ville se trouvent les traces de son passé, de son histoire, surtout dans son apparence et son architecture. Ainsi, chaque ville est un système hétérogène qui est créé par les diverses strates correspondantes aux différents siècles. D'après Roncayolo, "ce sont les temps différents de la ville qui sont mis au présent." (143). Selon Westphal, le présent est "un assemblage d'instant-points de forces hétérogènes, ceuxci sont autonomes." (226). Ainsi Jauss compare cette relation à une 
apparente simultanéité des étoiles dans le ciel d'aujourd'hui qui se décompose pour l'astronome en une immense diversité dans l'éloignement temporel. » (76).

Outre l'histoire de la ville, les mœurs et les coutumes passées de la société iranienne dans les différents domaines culturel, anthropologique et religieux sont le sujet de l'approche géocritique. Loti, en parlant du quartier de la nouvelle Djoulfa ou le faubourg arménien, explique ainsi l'histoire de ce quartier :

Et ces humbles quartiers, pour la plupart en ruines, où grouille une population pauvre, représentent les restes de la Djoulfa qui connut tant d'opulence à la fin du XVI ${ }^{\mathrm{e}}$ siècle, sous Chah-Abbas. (On sait comment ce grand empereur, — par des procédés un peu violents, il est vrai, - avait fait venir de ses frontières du Nord toute une colonie arménienne pour l'implanter aux portes de la capitale, mais l'avait ensuite comblée de privilèges, si bien que ce faubourg commerçant devint une source de richesse pour l'Empire. (110)

Le paysage pour Bouvier se déploie aussi avec une profondeur de durée qui marque une sorte de verticalité dans le temps. Il parvient à montrer une chronologie du souvenir, du passé glorieux de l'Iran qui se manifeste par une teneur archéologique :

Au XVII e siccle, avec six cent mille habitants, Ispahan était capitale d'empire et l'une des villes les plus peuplées du monde. Elle n'en a plus aujourd'hui que deux cent mille. Elle est devenue «province », elle s'est rétrécie, et ses immenses et gracieux monuments séfévides flottent sur elle comme des vêtements devenus trop grands. (Bouvier 636-637)

Les deux descriptions se superposent et permettent une analyse parallèle des paysages d'Ispahan qui montre cette hétérogénéité de pensée chez ces deux voyageurs occidentaux. À la suite de ses descriptions sur le quartier de la Nouvelle Djolfa, Loti présente un résumé de son historique et de ses habitants.

En fait, tous les monuments d'Ispahan datent de l'époque de ce roi : la Place Impériale (Maïdan Chah), les bazars, la Mosquée Impériale (Masjed-é Chah), le palais de Chah Abbâs, Tscharbag et tous les autres monuments qui se trouvent dans la Place Impériale qui se nomme la Place d'Imâm ou Maïdan-é Emâm aujourd'hui :

De même que Chiraz était la ville de Kerim-Khan, Ispahan est la ville de ChahAbbas. Avec cette facilité qu'ont eue de tout temps les souverains de la Perse à changer de capitale, ce prince, vers l'an1565, décida d'établir ici sa cour, et de faire de cette ville, déjà si vieille et du reste à peu près anéantie depuis le passage éffrayant de Tamerlan, quelque chose qui étonnerait le monde. (121)

La trace du passé de la ville se reflète aussi dans les magnifiques mosaïques et faïences. L'avenue de Tscharbag est d'après Loti l'une des «majestueuses allées » construites sous l'ordre de Chah Abbâs. Il la nomme «les Champs-Elysées d'Ispahan.» (126). Après la description de cette 
avenue, il compare le Roi-Soleil, Louis XIV, le roi de la France qui est contemporain de Chah Abbâs, avec ce dernier :

À l'époque où resplendissait chez nous la cour du Roi-Soleil, la cour des Chahs de Perse était sa seule rivale en magnificence; Ispahan, près d'être investie par les barbares de l'Est, atteignait l'apogée de son luxe, de ses raffinements de parure, et le Tscharbag était un rendez-vous d'élégances telles que Versailles même n'en dut point connaître. (ibid.)

Même l'architecture d'un palais comme «le palais des miroirs » ou «le palais des quarante colonnes » rappellent un temps plus ancien, celui des Achéménides : «Pour nos yeux, ce palais a l'étrangeté de lignes et la sveltesse outrée de l'architecture achéménide. » (ibid.)

Dans les bazars d'Ispahan, il y a même les nefs du grand empereur où « on voit son image démultipliée : le Chah-Abbas, avec sa longue barbe qui pend jusqu'à la ceinture, rendant la justice, le Chah-Abbas à la chasse, le Chah-Abbas à la guerre, partout le Chah-Abbas. » (141). Bouvier a aussi intégré le temps passé à sa description de l'Ispahan actuel. Selon lui, ces monuments ne sont pas solides et sont en train de se détériorer. Il les a comparés aux «vêtements devenus trop grands » (641) pour cette ville. Il trace alors un panorama de l'Ispahan d'autrefois et de l'Ispahan actuel. Mais, comme exprime Jaton : «Bouvier s'arrête en réalité sur un présent fugace, sur des instants toujours changeants plutôt que sur une reconstruction des strates qui ont servi à édifier l'univers qu'il traverse. » (13)

Outre l'historique de la ville qui révèle la coïncidence du présent et du passé, Loti et Bouvier parlent également de la culture iranienne et notamment du peuple d'Ispahan.

Malgré l'avis de la plupart des voyageurs qui ont été attirés par l'hospitalité des Ispahaniens, Loti parle de leur inhospitalité :

On aurait pu, en forçant un peu l'étape, arriver enfin ce soir à Ispahan; mais la tombée de la nuit nous a paru un mauvais moment pour aborder une ville où l'hospitalité est problématique, et nous avons décidé de nous arrêter dans un caravansérail, à trois lieues des murs. (Loti 107)

Lorsqu'il arrive au quartier du Djoulfa, là, les Arméniens lui déclarent que les chrétiens n'ont pas droit de loger dans le centre d'Ispahan. Mais il quitte ce quartier et va vers le centre-ville. Il refuse l'invitation des Arméniens à rester dans leurs maisons, car il tient « à habiter la belle ville bleue ». Il ajoute ainsi : les gens de Djoulfa « regardent nos préparatifs de départ avec des airs narquois, des airs de dire : on les mettra à la porte et ils nous reviendront. » (111). Pourtant, Bouvier parle de l'hospitalité des Persans. En fait, lui et son compagnon étaient invités chez les cousins de leurs amis de Téhéran et ceux-ci leur avaient donné une adresse : «Les Persans sont hospitaliers, c'est entendu, mais il est tard et nous tombons mal. » Le maître de la maison est un commerçant de bazar et sa maison est selon Bouvier une «maison accueillante. » (632) 
Mais quand il parle des gens d'Ispahan, son avis change :

Sous ce dôme et devant les palais de la place, passent les Ispahani, hors d'échelle, affables, pas trop épris de franchise, avec cet air qu'ont si souvent les habitants des villes d'art, d'être jury dans un concours auquel l'étranger, quoi qu'il fasse, ne comprendra jamais rien. (638-639)

Intéressés par la religion et les coutumes islamiques des habitants de cette ville, les deux voyageurs parlent des lois religieuses tirées de ces convictions qui ont influencé la vie quotidienne. Les mosquées de cette ville, en tant que symboles islamiques, distinguent une zone bien précise pour les habitants musulmans qui est interdite aux chrétiens. C'est pourquoi les Arméniens habitent le faubourg arménien (Djoulfa) : «Ici, ce n'est que le faubourg arménien, le faubourg profane où les étrangers à l'Islam ont le droit d'habiter. » (Loti 110)

De plus, il y a une différenciation bien précise quant à la vie nocturne dans cette ville islamique. La défense de rester dehors pendant la nuit, est un élément qui marque ce décalage de culture : [...] la vieille capitale de Chah-Abbas n'a point de vie nocturne. Le portail de la maison du prince se ferme hermétiquement dès qu'il commence à faire noir. Les vieilles portes bardées de fer, qui séparent les uns des autres les différents quartiers, se ferment aussi partout; l'inextricable labyrinthe de la ville, où l'obscurité sera bientôt souveraine, se divise en une infinité de parties closes qui, jusqu'au retour du soleil, ne communiqueront plus ensemble : le suaire de plomb de l'Islam retombe sur Ispahan (125).

Loti mentionne à plusieurs reprises les «appels chantés des muezzins » (ibid.) : "Avant la tombée de la nuit, un peu de temps me reste pour faire station sur la grande place, où l'heure religieuse du Maghreb s'accompagne d'un cérémonial très antérieur à l'Islam et remontant à la primitive religion des Mages. » (132). Ici, il s'agit d'une stratification qui se fait en spirale. Par conséquent, les mêmes attitudes se répètent. Ici, les traces du passé lointain se manifestent de plus en plus dans le présent. La culture passée du zoroastrisme n'a pas disparu, mais elle est réinvestie dans la culture actuelle en évoquant l'annonce de la prière par les muezzins dans l'Ispahan d'aujourd'hui :

Après mes courses matinales, je suis toujours rentré pour l'instant où les muezzins appellent à la prière du milieu du jour (midi, ou peu s'en faut). A Ispahan, ce sont les muezzins qui donnent l'heure, comme chez nous la sonnerie des horloges, et ils chantent sur des notes graves, inusitées en tout autre pays d'Islam. (135-136)

Bouvier, tout en décrivant « une Province sans vin, ni vantardises ni voix de femmes ", (642) mentionne son désintérêt pour ces coutumes et s'étonne de certaines superstitions qui marquent la trace de cette fermeté dans la vie quotidienne.

Le vendredi, le jour férié et saint des habitants d'Ispahan, décrit par les deux auteurs-voyageurs, est un retour vers la nature. Les musulmans vont à la promenade :

Vendredi aujourd'hui, dimanche à la musulmane; il faut aller dans les champs pour faire comme tout le monde. [...] Ispahan se dépeuple aujourd'hui, déverse dans son oasis tout ce qui lui reste d'êtres vivants parmi ses ruines. (Loti 139) 
Bouvier a aussi la chance d'être présent dans une réunion familiale qui évoque ce que les habitants d'Ispahan font ce jour-là, une activité pleine de joie et de divertissements :

C'est vendredi, soir des réunions familiales, et la maison est pleine d'enfants, de parents de province qui vont et viennent en pyjama, grignotent des abricots secs, jouent au jacquet, transportent des couvertures, des lampes, des moustiquaires. (633)

C'est ici qu'il va comprendre chez les Iraniens le profond respect pour la famille et la raison de l'existence d'une grande amitié entre les gens, ce qui était complètement différent chez lui à cette époque.

\section{4) Espace, texte; le poids de l'intertextualité}

Tout énoncé d'un texte contient un ou plusieurs discours extraits d'autrui. C'est ainsi que la question de l'intertextualité se pose. Selon Riffaterre, « il suffit pour qu'il y ait intertexte que le lecteur fasse nécessairement le rapprochement entre l'auteur et ses prédécesseurs » (137). Ainsi, le lecteur entend la voix des autres à travers la voix de l'auteur dont il est en train de lire le texte. Alors, un texte est polyphonique. Une conception de l'intertextualité est une sorte d'interaction textuelle qui permet de considérer «les différentes séquences (ou codes) d'une structure textuelle précise comme autant de transformes de séquences (codes) prises à d'autres textes. L'intertextualité est une notion qui sera l'indice de la façon dont un texte lit l'histoire et s'insère en elle. » (Kristeva 311)

Quant à nos deux auteurs-voyageurs, l'aspect intertextuel apparait à travers l'influence des récits de voyage écrits durant les siècles précédents ou celle des ouvrages d'autres écrivains français. Par exemple, le premier mot de l'ouvrage de Nicolas Bouvier a une signification intertextuelle. Ce mot est choisi du premier quatrain du célèbre sonnet de Du Bellay qui s'intitule Heureux qui comme Ulysse. Là, ce mot contient un ensemble de valeurs et de comportements éthiques, issus des expériences face à d'autres cultures. Ainsi, Bouvier transmet dès le début de son ouvrage le but de son voyage en recourant au poème de Du Bellay. De plus, on constate que ce mot est également hérité de Montaigne. (Jaton 5)

Mais quelle place occupent-ils les autres écrivains dans le texte cible? Barthes écrit :

Lisant un texte rapporté par Stendhal (mais qui n'est pas de lui) j'y retrouve Proust par un détail minuscule [...]. Je comprends que l'œuvre de Proust est, du moins pour moi, l'œuvre de référence [...]. Et c'est bien cela l'inter-texte : l'impossibilité de vivre hors du texte infini, que ce texte soit Proust, ou le journal quotidien, ou l'écran télévisuel : le livre fait le sens, le sens fait la vie (58-59).

Ou comme il le déclare: «Le texte ne vit qu'en contact avec un autre texte $»(232)$. 
Pourtant, les lectures des voyageurs influencent leurs ouvrages et on y trouve la même trace chez Loti. On y aperçoit celle du Voyage en Perse de Jean Chardin quand il peint la place Impériale comme : «[...] la merveille de la ville, et dont s'ébahirent tant, au XVII' siècle, les premiers Européens admis à pénétrer ici » (118-119), ou le palais de Zelleh-Sultan comme le « dépôt des vins, que Chardin, au XVII e siècle, nous décrivit comme tout rempli de coupes et de carafons en "cristal de Venise, en porphyre, en jade, en corail, en pierre précieuse." (135). Dans la description de Bouvier de cette ville, on ne constate aucune phrase qui montre cette démarche intertextuelle, on dirait qu'il n'avait pas intérêt pour le passé lointain de l’Iran.

Pourtant, dans les deux ouvrages, nous trouvons des passages où nos voyageurs font référence à leur pays d'origine, la France, ou aux pays européens. Dans ces passages, ils comparent ce qu'ils voient des monuments, des mœurs et de la nature de cette ville et de ses habitants avec les pays européens, surtout la France.

Dans la plupart des descriptions, cette vision intertextuelle se manifeste également par un regard comparatif chez nos deux voyageurs dont l'exemple se trouve aussi chez Bouvier quand il mentionne les similitudes entre la Mosquée royale et Notre-Dame de Paris (634). Il y parle sur les traces du passé dans le présent en donnant les informations historiques sur ces deux monuments qui ont montré chacun à son tour la majesté d'une civilisation.

\section{Conclusion}

En se référant à ces deux récits de voyage, on a constaté un lien étroit entre la littérature et la géographie. Ce lien a permis à nos auteurs-voyageurs de connaître autrui et de découvrir sa culture, son histoire et même un nouveau monde féerique et imaginaire à travers l'architecture des monuments de la ville d'Ispahan, décrite dans leurs récits de voyage. Pourtant, Loti et Bouvier n'ont pas pu se débarrasser de leurs mentalités ainsi que de leurs souvenirs de la vie occidentale et ils ont mêlé parfois l'image de ce qu'ils ont vu à celle de leur propre pays.

En appliquant les quatre éléments de la géocritique aux récits de voyage de Pierre Loti et de Nicolas Bouvier, on fait face à une multiplicité de focalisations qui se manifestent dans les descriptions des lieux données. Loti a bien réussi à évoquer tous les détails de cette ville visitée à la fin du XIX ${ }^{e}$ siècle et à mettre en relation l'imaginaire et l'espace réel, tandis que Bouvier a essayé de donner une description sommaire et moins attentive de cette ville orientale. Profitant de plusieurs aspects sensoriels, ces deux écrivains contribuent, selon la règle de la polysensorialité, à la construction d'une image spéciale de différentes parties de cette ville. 
Ispahan présenté chez Loti est une ville féerique et orientale où ses sens visuel, auditif et olfactif l'aident à présenter cette ville sous tous ses aspects. Elle représente le stéréotype d'un "Orient merveilleux" à la manière de Montesquieu dans ses Lettres persanes, qui va restituer l'image donnée par les grands voyageurs du XVII ${ }^{\mathrm{e}}$ siècle. Bouvier profite de ses sens, notamment la vue, dans ses observations, mais l'Ispahan chez lui est imprégnée du sentiment de la mort, qui ne lui donne pas le sentiment du réel et il trouve absurde sa présence en ce lieu.

La nostalgie et la trace du passé lointain d'Ispahan dans son actualité, marquée dans le domaine de la stratigraphie, se reflètent presque dans toutes ces descriptions. Alors, la ville ancienne est cachée sous la ville actuelle et les strates du temps passé sont visibles à travers les monuments et les coutumes de cette ville.

Pour conclure, l'Ispahan de Loti est plus magique et désirable, adaptable au XIX ${ }^{\mathrm{e}}$ siècle français, siècle des romantiques passionnés par l'Ailleurs et par l'exotisme. En revanche, l'Ispahan de Bouvier est le symbole d'une société en train de plonger dans un monde dépourvu de la sensibilité et de grâce où la guerre et les changements sociaux occupent une place primordiale dans l'éloignement des hommes de leurs origines. L'écriture de Bouvier, qui est un mélange d'abondance et d'austérité dans certains cas, montre un aspect inconnu qui ne doit pas être négligé; le mélange du sentiment de l'absurde et de ruine face aux attraits fascinants de cette "moitié du monde", montre chez lui l'aspect intouchable de cette ville. 


\section{Bibliographie}

Bachelard, Gaston. Poétique de l'espace. PUF, 1957.

Bakhtine, Mikhail, et Valentin Nikolaevic Vološinov. Le Marxisme et la philosophie du langage. Minuit, 1977.

Barthes, Roland. Le Plaisir du texte. Seuil, 1973.

Bischoff, Liouba, "La géographie précaire de L’Usage du monde", Viatica, Bouvier, intermédiaire capital, 2017.

Bouvier, Nicolas. L'Usage du monde. Julliard, 1964.

Cogez, Gérard. Les Écrivains voyageurs au XXe siècle. Seuil, 2004.

Jaton, Anne-Marie. "Nicolas Bouvier paradoxal". Revue des littératures et des Arts, No17, 2017.

Jauss, Hans-Robert. Pour une esthétique de la réception. Gallimard, 1978.

Kristeva, Julia. "Problème de la structuration du texte", Théorie d'ensemble, Seuil, 1968, pp.297-315.

Loti, Pierre. Vers Ispahan. Bibebook, Bibliothèque Nationale de France, 1904.

Outeirinho, Marie de Fátima. "Les apports réflexifs d'une géographie littéraire pour la littérature de voyage". Cadernos de literatura comparada, no. 33, Université de Porto, 2015, pp.149-159.

Perrault, Charles. Contes. Édition établie par Gilbert Rouger, Classiques Garnier, 2014.

Riffaterre, Michael. "Sémiotique intertextuelle : l'interprétant in Rhétoriques. Sémiotiques". Revue d'Esthétique, vol. 5, collection 10/18, no. 1-2, 1979, pp. 128-150.

Roncayolo, Marcel. La Ville et ses territoires. Gallimard, 1990.

Rostkowski, Joëlle. "Voyage à Ispahan". Catalogue de l'exposition, Orenda Art International, Paris, 2015.

Tuan, Yi-Fu. Space and place: The perspective of experience. University of Minnesota Press, 1977.

Westphal, Bertrand. La Géocritique, mode d'emploi. PULIM, 2000.

---. "Pourquoi une géocritique de Lisbonne?", Lisbonne : Géocritique d'une ville, Etudes réunies par Alain Montandon, Presses universitaires Blaise Pascal, 2006, pp. 7-20.

---. La Géocritique. Réel, fiction, espace. Minuit, 2007. 